\title{
Evaluación de la Administración Infiltrativa de vitamina E y alendronato en la Reparación de Alvéolos Post Exodoncia de Ratas
}

\author{
Evaluation of the Infiltrative Administration of Vitamin E and \\ Alendronate in the Repair of Alveoli Post-Extraction in Rats
}

Cecilia Moro; Alejandra Aguzzi \& Carolina Virga

MORO, C.; AGUZZI, A. \& VIRGA, C. Evaluación de la administración infiltrativa de Vitamina E y Alendronato en la reparación de alvéolos post exodoncia de ratas. Int. J. Odontostomat., 13(4):402-410, 2019.

RESUMEN: Los bisfosfonatos (BP) disminuyen la resorción ósea al frenar la actividad de los osteoclastos. La vitamina $\mathrm{E}$ es antioxidante y su efecto positivo en el hueso sería mediante la prevención del estrés oxidativo. Se estudió la administración infiltrativa de Alendronato y Vitamina E para determinar si favorecían la formación de hueso en la reparación ósea del alvéolo postexodoncia. Se utilizaron ratas machos Wistar ( $\mathrm{n}=96)$, de $90 \pm 15 \mathrm{~g}$, se les realizó la exodoncia de los primeros molares inferiores. Fueron dividos en 4 grupos: Un grupo control (C) recibió solución salina. El grupo AL 0,5 mg/ $\mathrm{Kg}$; grupo E recibió $20 \mathrm{mg} / \mathrm{kg}$; y grupo con tratamiento combinado AL y E. Los animales se sacrificaron a los $0,7,15$ y 30 días postextracción. Se realizó la resección de las mandíbulas; las muestras fueron descalcificadas con EDTA y luego se incluyeron en parafina. Se realizaron cortes histológicos y se colorearon con Hematoxilina/Eosina. Se realizó análisis histológico e histomorfométrico. Se utilizó análisis de Varianza (ANOVA). En el análisis histológico, a los 7 y 15 días el grupo E presentó mayor neoformación de tejido óseo que los otros grupos. A los 30 días se observó hueso maduro con presencia de osteonas en el grupo E. En el estudio histomorfométrico a los 15 y 30 días se evidencian diferencias significativas en el número de osteoblastos por mm lineal, entre el grupo $A L+E$ y $C(p<0,01)$ y a los 30 días se encontró diferencia entre el grupo $E$ y $C(p<0,01)$. Al medir espesor trabecular se observó a los 30 días diferencias significativas entre el grupo $A L+E$ y $C(p<0,01)$ y entre el grupo $C$ y $E(p<0,01)$. La Vitamina $E$ demostró que administrada por vía infiltrativa favorece la remodelación ósea en los alvéolos post exodoncia.

PALABRAS CLAVE: osteogénesis, alendronato, vitamina E.

\section{INTRODUCCIÓN}

La extracción dental es uno de los procedimientos terapéuticos más frecuentes en Odontología; el hueso alveolar es un tejido dependiente del diente al que sostiene y se desarrolla cuando éstos erupcionan. Con la exodoncia, el proceso alveolar se atrofia, debido a la pérdida de su función (Aimetti et al., 2009). Por lo tanto, al mismo tiempo se produce la curación y la remodelación de los tejidos duros y blandos del alvéolo post-extracción, que continúa incluso después de que se rellene el alvéolo de hueso neoformado (Barone et al., 2013).

La cicatrización de un hueso largo fracturado se produce por osificación endocondral, pero la curación del alvéolo después de la extracción del diente se produce sin formación histológica de cartílago (Ting et al., 1993). Diversos estudios observaron que inmediatamente después de la extracción dentaria en ratas el coágulo de sangre rellena el alvéolo y la membrana periodontal se encuentra unida al hueso. Se observaron trabéculas de hueso a los 7 días en el fondo de la cavidad y en el tercio apical de la superficie interna de las paredes vestibulares y linguales. A los 14 días, las superficies trabeculares mostraron intensa actividad ósea; mientras que a los 30 días, el alvéolo se encontraba relleno por hueso reticular. Los osteoblastos activos se observaron principalmente en la cresta alveolar. Al cabo de los 60 días, el alvéolo completo se rellenó con tejido óseo laminar (Guglielmotti et al., 1985; Devlin et al., 1997). 
Los bisfosfonatos (BP) se unen rápidamente al hueso, inhiben la transformación del fosfato de calcio amorfo en hidroxiapatita y dificultan el crecimiento de los cristales de ésta. Además, reducen la velocidad de disolución de dichos cristales. Pero su efecto fundamental estriba en su capacidad para disminuir la resorción ósea al frenar la actividad y la supervivencia de los osteoclastos (Thompson et al., 2006). Durante el proceso de resorción se liberan moléculas del BP desde la fase mineral del hueso, las cuales posteriormente son internalizadas por el osteoclasto. El pH ácido de la laguna de resorción contribuye en este proceso, dado que determina la disociación del BP de la hidroxiapatita (Rogers, 2003).

El a-tocoferol fue el primer análogo de la vitamina $E$ a ser reconocido, funciona como un antioxidante que rompe la cadena que interrumpe la propagación de especies reactivas de oxígeno a través de las membranas lipídicas mediante la depuración de los radicales peroxilo lipídico. Los radicales libres suprimen la diferenciación osteoblástica y facilitan la resorción ósea mediante la activación de osteoclastos (Azzi et al., 2003). Como tal, el estrés oxidativo se ha sugerido para crear un desequilibrio entre la osteogénesis y la resorción ósea. Los efectos positivos de la vitamina $E$ en el hueso mediante la prevención del estrés oxidativo podrían ser mediados a través de una vía similar que implica el Receptor activador del factor nuclear Kappa B (RANK) / Ligando del RANK (RANKL). Diversos estudios mostraron que la vitamina $E$ evitó la osteoclastogénesis y la resorción ósea mediante la supresión de la expresión y señalización de RAKL sin afectar a la expresión de Osteoprotegerina (OPG) (Ha et al., 2011; Muhammad et al., 2012).

Por las acciones anteriormente expresadas, se estudió la administración infiltrativa de Alendronato y Vitamina E para determinar si favorecían el aumento de la formación de hueso en la regeneración ósea del alvéolo post exodoncia.

\section{MATERIAL Y MÉTODO}

Preparación de las formulaciones: Se preparó una solución de Alendronato para ser aplicada semanalmente por vía submucosa profunda (infiltrativa) con una dosificación de $0,5 \mathrm{mg} / \mathrm{Kg}$ de peso corporal. Vitamina $E$ se administró tres veces por semana también por vía submucosa profunda con una dosificación de $20 \mathrm{mg} / \mathrm{kg}$ de peso corporal. Ambas drogas fueron administradas siempre en el mismo horario (10:00 AM).
Prueba en animales de experimentación: El presente estudio experimental in vivo fue aprobado por el Comité Institucional para el Cuidado y Uso de Animales de Laboratorio, y el manejo de los animales fue realizado de acuerdo a las normas establecidas por SECyT siguiendo las normativas de éste Comité (Res. HCD 674/09 Facultad de Ciencias Médicas). Se utilizaron 96 ratas macho de la línea Wistar, por ser éste un animal reproducible y de fácil mantenimiento (AliErdem et al., 2011). Se tuvo especial cuidado en que el peso de los animales fuera de $90 \pm 15 \mathrm{~g}$, debido a que en ratas con pesos superiores se produce una cementosis radicular que dificulta la extracción dentaria. Los animales se dividieron en 4 grupos de 24 ratas cada uno. Un grupo fue control (C) y recibió semanalmente $1 \mathrm{ml} / 100 \mathrm{~g}$ de peso corporal de solución salina por vía infiltrativa inyectada con una jeringa de tuberculina de $1 \mathrm{ml}$ en el fondo de surco a la altura del primer molar inferior (previa aplicación de Xilazina para lograr relajación muscular y facilitar la manipulación). El segundo grupo Alendronato $(A L)$ recibió semanalmente $0,5 \mathrm{mg}$ de $\mathrm{AL} / \mathrm{Kg}$ de peso corporal por vía infiltrativa. El tercer grupo Vitamina $\mathrm{E}(\mathrm{E})$ recibió tres veces por semana $20 \mathrm{mg} / \mathrm{kg}$ de peso corporal por la misma vía. El cuarto grupo recibió tratamiento combinado con AL y E por vía infiltrativa.

Al inicio del experimento los animales fueron anestesiados con una solución de ketamina/xilazina en una relación $8 \mathrm{mg} / 1,28 \mathrm{mg}$ respectivamente por cada $100 \mathrm{~g}$ de peso corporal. A los fines de lograr el abordaje y realizar maniobras quirúrgicas en la cavidad bucal de la rata, se tomaron como referencia trabajos publicados anteriormente (Guglielmotti et al.; Fontana, 2009) y se preparó una camilla especial que permite mantener a los animales en posición decúbito dorsal a boca abierta mediante un sistema de ligaduras. Una vez anestesiadas, se colocaron las ratas sobre la camilla y, previa asepsia del campo quirúrgico con iodopovidona, con instrumental adecuado al volumen y forma de los elementos dentarios se procedió a realizar la extracción de los primeros molares inferiores. El alvéolo no fue rellenado con ningún material sino sólo por sus propios coágulos. Al finalizar el experimento se realizó la eutanasia de los animales mediante inyección intracardíaca de cloruro de potasio, bajo anestesia general. Los sacrificios para la toma de muestras fueron a los $0,7,15$ y 30 días.

Estudios histológicos e histomorfométricos: Luego de la eutanasia de los animales se realizó la resección de las mandíbulas, las cuales fueron colocadas en frasco estéril y fijadas en formaldehido al $10 \%$, 
posteriormente fueron descalcificadas con EDTA a pH 7 y $4{ }^{\circ} \mathrm{C}$ durante 60 días y luego incluidas en parafina. Se realizaron cortes histológicos a nivel de los alvéolos en sentido longitudinal utilizando la raíz mesial del mismo por ser esta la de mayor tamaño. Las muestras fueron coloreadas con Hematoxilina/Eosina (H/E) para el análisis histopatológico e histomorfométrico correspondiente, seleccionando el tercio medio del centro del alveolo.

Los cortes fueron observados por microscopía óptica y analizados a un aumento de 40x 100x y 400x para su valoración cualitativa. Las muestras se analizaron histomorfometricamente mediante un programa de análisis de imágenes digitales sobre microfotografías de cortes histológicos del tejido óseo (Image Pro-Plus 4,5) del Área de Biología Oral (ABO), Facultad de Odontología de la Universidad Nacional de Córdoba. Este procedimiento se llevó a cabo en cada grupo y tiempo experimental. A partir de este análisis, se estableció un área llamada 4, como la zona representativa a estudiar, al estar ubicada en el centro del alveolo post-extracción. El análisis histomorfométrico se realizó utilizando microfotografías de 100x, con el programa Image-Pro Plus v4.5.0.29 obtenidos de 5 cortes histológicos de cada grupo (Weibel et al., 1966). Así mismo se realizaron las siguientes mediciones: 1) Medición de la Regeneración Ósea Alveolar Post-extracción; 2) Medición de espesor trabecular; 3) Cantidad de osteocitos por $\mathrm{mm}^{2}$; 4) Cantidad de osteoblastos; 5) Medición de zonas de resorción.

Estudios Estadísticos: La comparación de los datos se llevó a cabo mediante contrastes paramétricos y no paramétricos. En aquellos casos en los cuales se cumplían los requisitos de normalidad y homogenei-

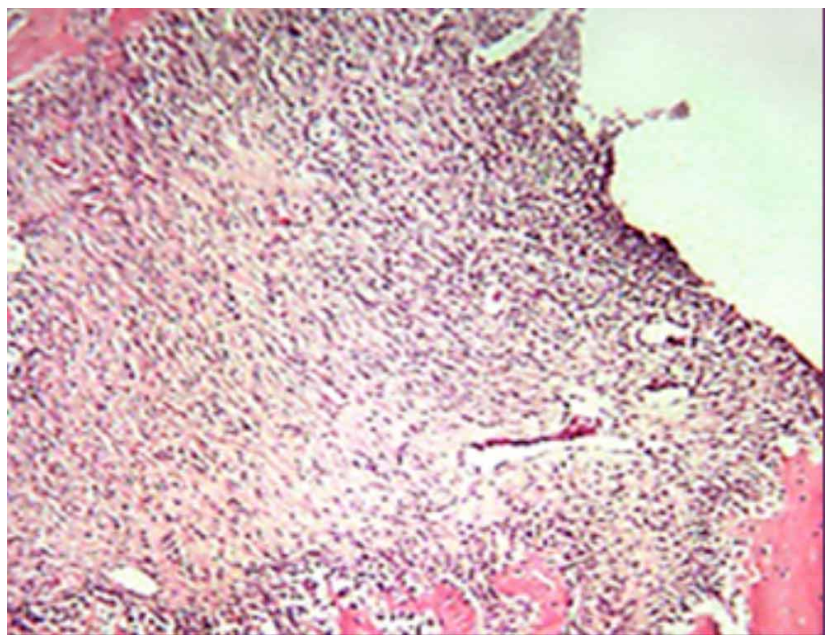

Fig. 1. Imagen histológica Control día 7. 100 x HE. dad, se realizó un análisis de la Varianza (ANOVA) a dos criterios de clasificación (tratamientos: $\mathrm{C}, \mathrm{AL}, \mathrm{E}$, $A L+E$, y tiempos de tratamiento: 0, 7. 15 y 30 días). Se utilizó como test complementario la prueba de Tukey que realiza comparaciones múltiples entre categorías. Se consideraron diferencias significativas si $p<0,05$. Para el análisis de datos, construcción de gráficas y análisis estadísticos se utilizó el software (GraphPadPrism 2.0, GraphPad Software, San Diego, CA USA).

\section{RESULTADOS}

Análisis histológico descriptivo. A los 7 días, en el grupo $\mathrm{C}$, desde la zona superficial hacia el interior del alvéolo, se observó tejido de granulación y tejido fibroblástico sustituyendo al coágulo (Figs. 1 y 2). En el grupo AL, se distinguió, desde la superficie hacia el interior del alvéolo, presencia de escaso tejido de granulación y tejido fibroso en el resto del alvéolo. En el grupo E, se pudo apreciar la presencia de tejido de granulación en la zona más superficial del alvéolo, tejido fibroso y en la zona más apical un trabeculado óseo que no se aprecia en los demás grupos a los siete días (Figs. 3 y 4). En el grupo AL+E, hubo presencia de tejido de granulación y de tejido fibroso. En la zona apical y cercana a las tablas vestibular y lingual, algunos islotes pequeños de tejido óseo de neoformación.

A los 15 días, en el grupo C, comenzó la formación de hueso reticular, inmaduro y persisten islotes de tejido fibroso. En el grupo AL, se observó una red fibrosa organizada en el espacio alveolar y un

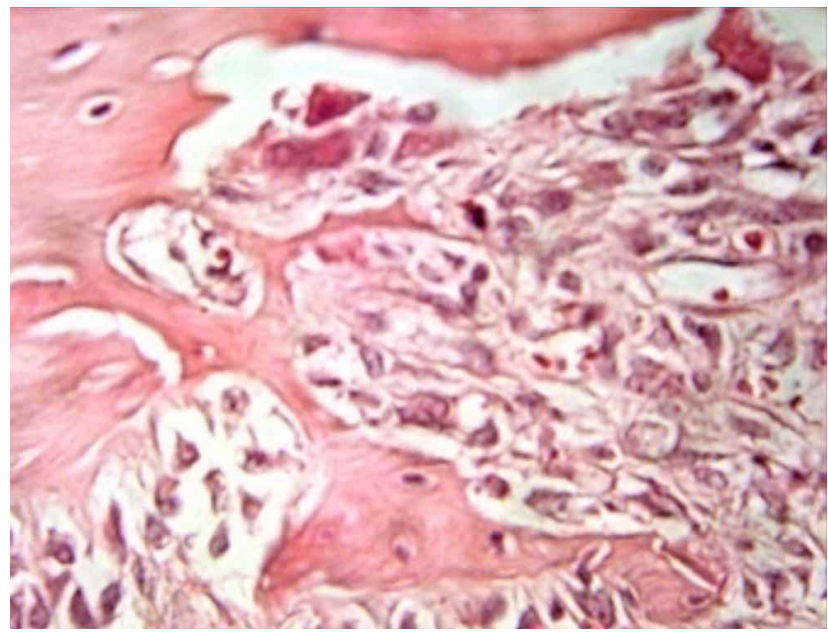

Fig. 2. Imagen histológica Control día 7. 400 x HE 


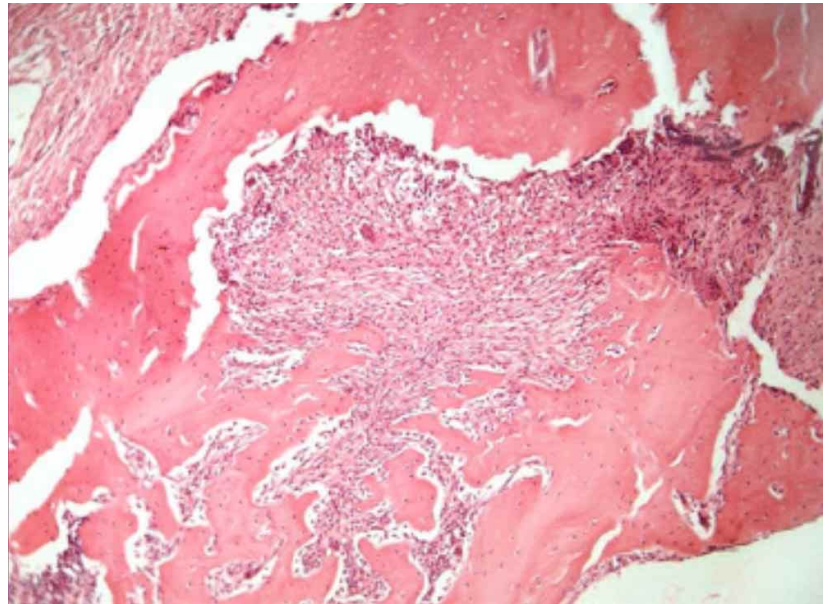

Fig. 3. Imagen histológica Vitamina E día 7. 100x HE.

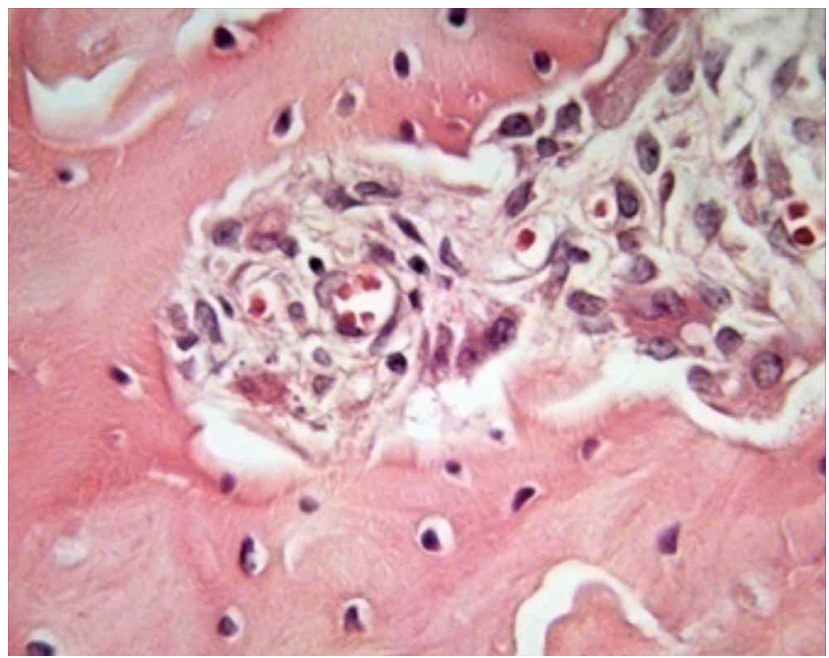

Fig. 4. Imagen histológica Vitamina E día 7. 400 x HE.

trabeculado óseo nuevo con presencia de tejido osteoide en algunos sectores. En el grupo E, se va reparando el sitio de la exodoncia con tejido óseo reticular con médula ósea inflamatoria y aún se observa una zona de fibrosis en la parte superficial. En superficie se apreció mayor volumen de hueso con respecto al grupo $C$. En el grupo $A L+E$, se observó la formación de hueso reticular y persistencia de tejido fibroso.

Finalmente, a los 30 días, el grupo C, presentó un trabeculado óseo de hueso reticular con espacios medulares, rodeado de ambas corticales (Figs. 5 y 6 ). En el grupo AL, la cavidad se encontró ocupada por tejido óseo reticular, con características de un trabeculado normal rodeado por ambas corticales. En el grupo E, el espacio se llenó con tejido óseo nuevo donde se pudo observar que el mismo se dispuso con una organización similar a osteonas o sistemas de

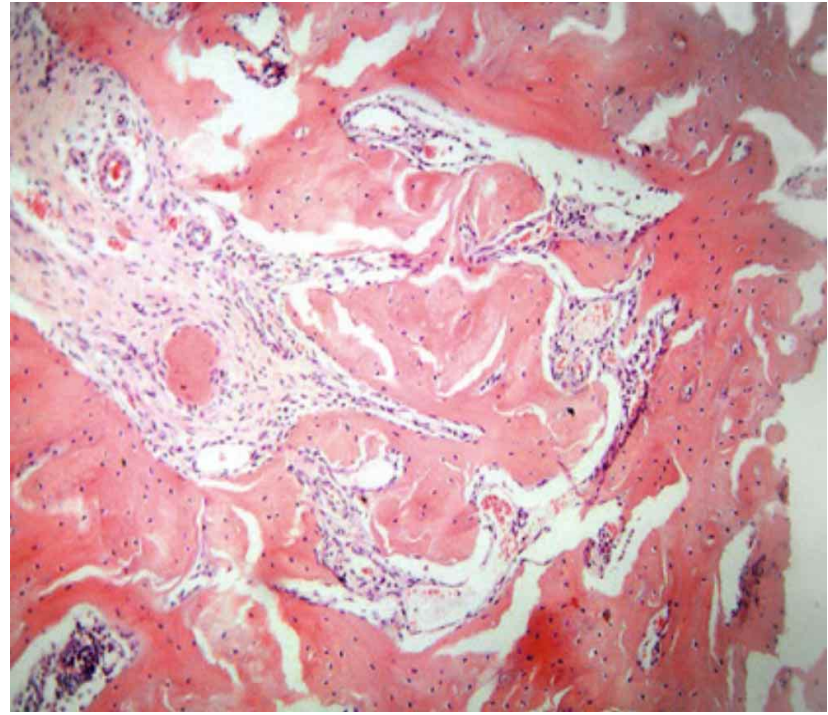

Fig. 5. Imagen histológica Control día 30. 100 x HE.

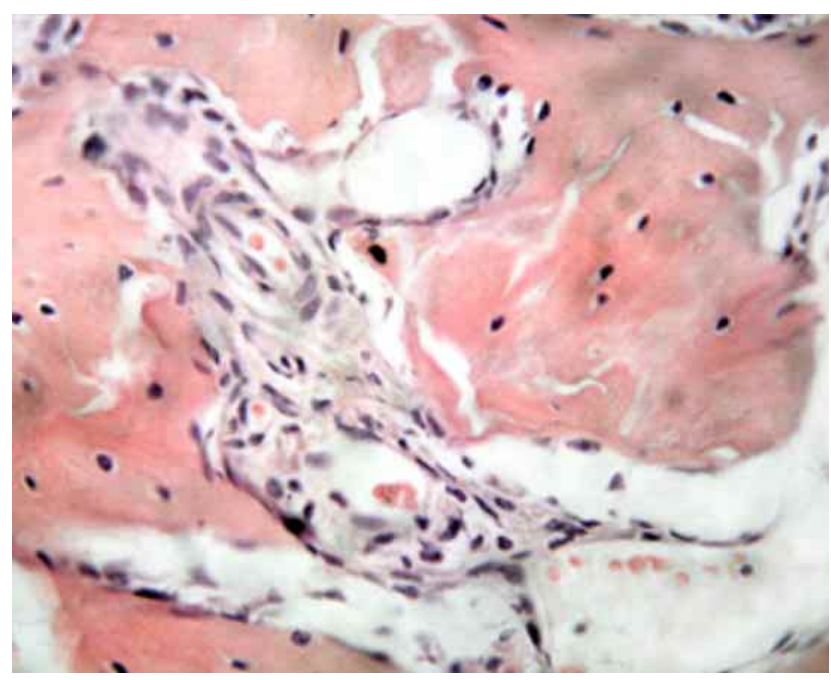

Fig. 6. Imagen histológica Control día 30. 400 x HE

havers, lo que haría referencia a un tipo de hueso más maduro (Figs. 7 y 8 ). Algo similar se apreció en el grupo de la combinación de drogas $(A L+E)$.

Estudios histomorfométricos. El análisis histomorfométrico se realizó según los parámetros detallados en Materiales y métodos. Los componentes analizados a los 7, 15 y 30 días fueron: Tejido de granulación, Tejido óseo neoformado y Médula ósea. Las diferencias de las áreas analizadas resultaron escasas a los 7 días. A los 30 días las diferencias se hacen evidentes entre los grupos $E$ y $C$, sin mostrar diferencias significativas. A los 15 días el grupo $\mathrm{E}$ registró valores de hueso neoformado por encima de todos los grupos, con diferencias significativas con el grupo $C(p<0,05)$ (Fig. 9). 


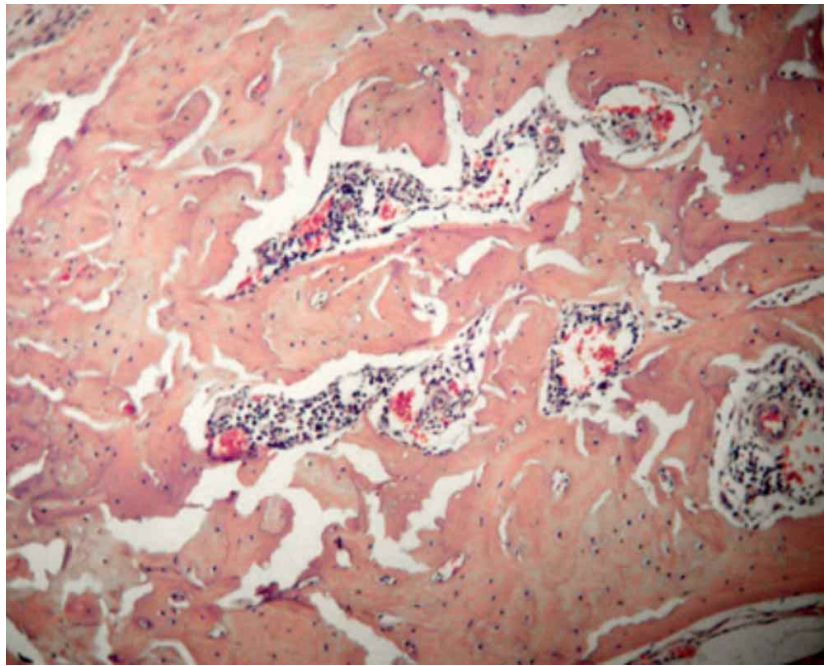

Fig. 7. Imagen histológica Vitamina E día 30. 100 x HE

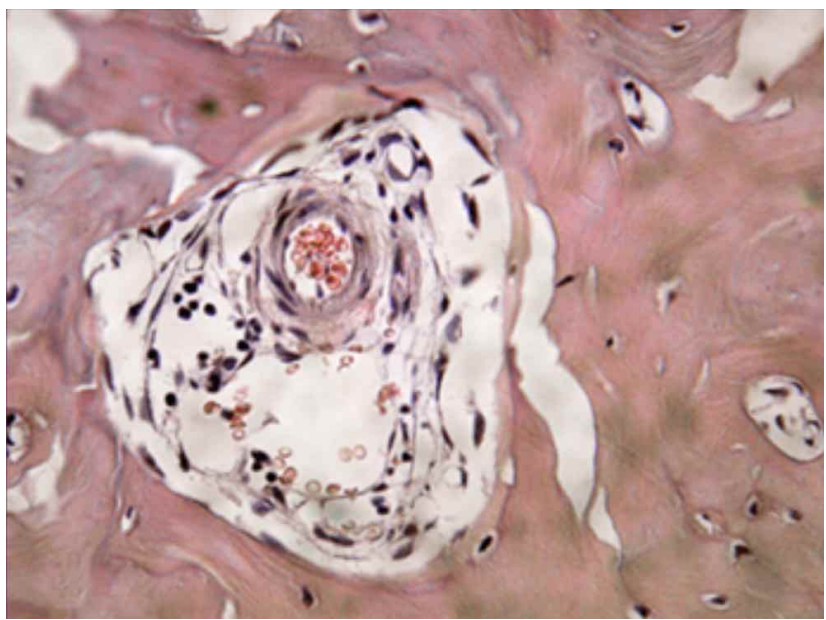

Fig. 8. Imagen histológica Vitamina E día 30. 400 x HE.
Las diferencias de espesor trabecular resultaron escasas en las primeras dos etapas evaluadas (7 y 15 días), pero a los 30 días se hacen evidentes, en esa etapa los grupos $\mathrm{E}$ y $\mathrm{AL}+\mathrm{E}$ registraron valores medios de espesor por encima de los grupos $\mathrm{C}$ y $\mathrm{AL}$, con diferencias significativas con el grupo $C(p<0,01)$ (Fig. 10). Al analizar la cantidad de osteocitos por $\mathrm{mm} 2$ en hueso trabecular, no se encontraron diferencias entre los grupos experimentales.

En cuanto a la cantidad de osteoblastos, el grupo AL+E comienza a diferenciarse del resto de los grupos ya en las primeras etapas, mostrando una marcada actividad osteoblástica. Recién a los 30 días el grupo (sin combinación con $\mathrm{AL}$ ) alcanza valores similares a $A L+E$; éstos grupos, registraron mayor actividad osteoblástica que los grupos AL y C en todas las etapas evaluadas, con diferencias significativas con respecto al C $(p<0,01)$ (Fig. 11).

Todos los grupos mostraron una tendencia similar, con mayor actividad osteoclástica en la etapa inicial que luego se estabiliza a partir de los 15 días y precisamente en este momento (15 días), los grupos $E$ con y $\sin A L$, muestran menor actividad que los otros dos (C y $\mathrm{AL}$ ) y a los 30 días el grupo $\mathrm{C}$ registró una media algo por encima del resto de los grupos. Más allá de la descripción de los valores de tendencia central (media porcentual de zonas con resorción), las diferencias entre grupos no resultaron significativas.

\section{5 días}

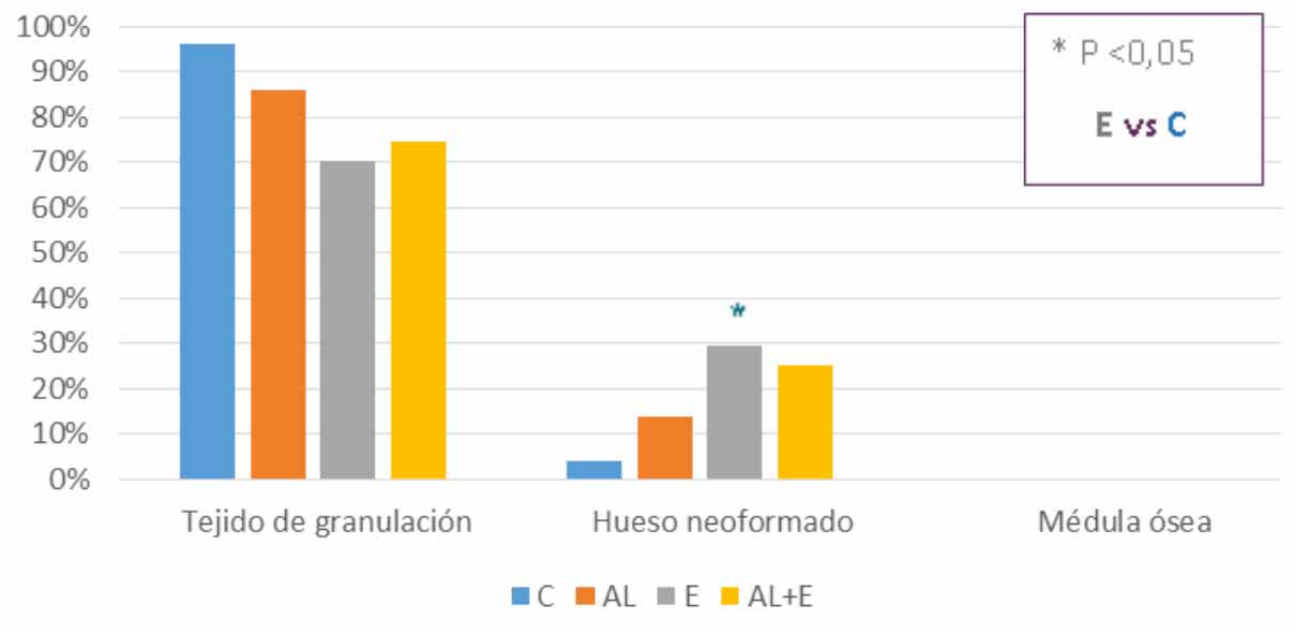

Fig. 9. Distribución esquemática de volumen y porcentajes de las áreas analizadas según grupo experimental a los 15 días del inicio del tratamiento. 


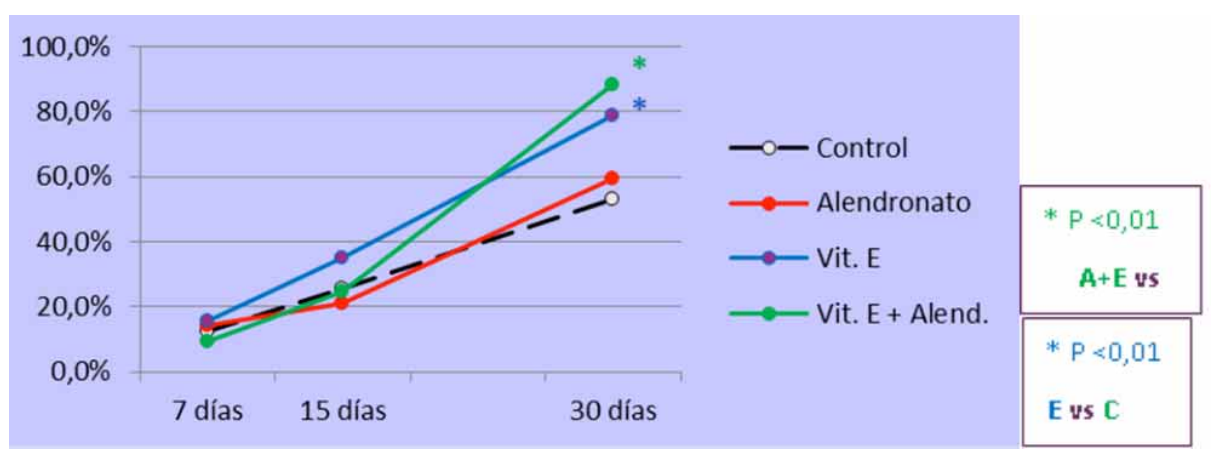

Fig. 10. Porcentaje de espesor óseo trabecular según etapa y grupo.

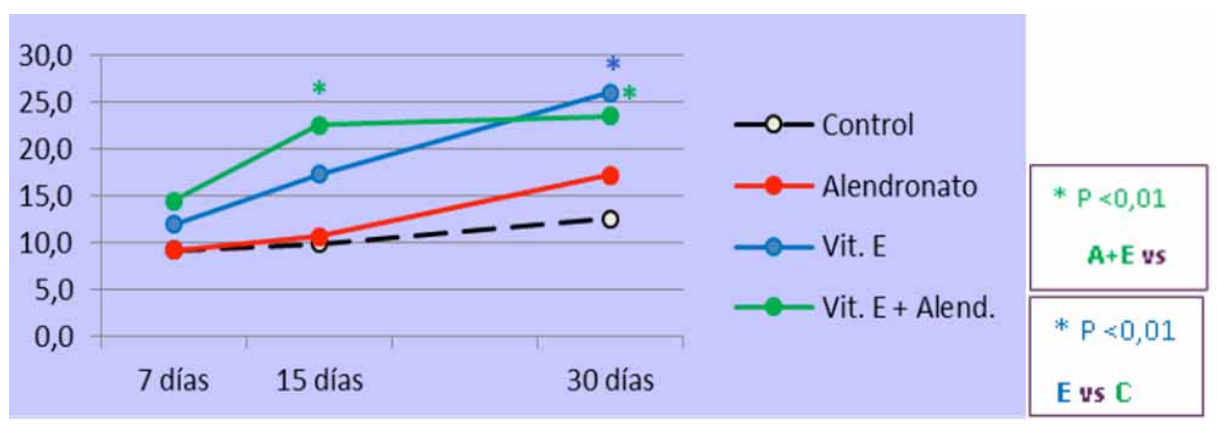

Fig. 11. Cantidad de osteoblastos por $\mathrm{mm}$ lineal según etapa y grupo.

\section{DISCUSIÓN}

En esta investigación, el análisis histológico del hueso permitió analizar la actividad celular osteoblástica y osteoclástica, y las características del hueso nuevo. Esto permitió determinar cómo se fue reparando el alvéolo postexodoncia en función del tiempo y el tratamiento utilizado. A los 7 días se pudo apreciar hueso reticular en el grupo $E$, que no se observó en los demás grupos. El grupo E, a los 15 días, muestra mayor volumen de hueso nuevo en el tercio medio y apical del alvéolo, con respecto a los demás grupos. Finalmente, a los 30 días, tanto $\mathrm{E}$ como $\mathrm{AL}+\mathrm{E}$ evidencian la presencia de hueso compuesto (Roberts et al., 1987) que tiene áreas de hueso reticular y áreas de hueso laminar con formación de osteonas o sistemas de Havers, lo que determina que Vitamina E mejora las condiciones óseas del alveolo en reparación.

Los parámetros histomorfométricos de remodelación ósea utilizados en este estudio se dividen en: medición de área de tejido de granulación, de tejido óseo neoformado y de médula ósea; espesor trabecular, cantidad de osteocitos por $\mathrm{mm}^{2}$ en hueso trabecular, cantidad de osteoblastos por $\mathrm{mm}$ lineal y cantidad de osteoclastos sobre trayecto perimetral de hueso trabecular en zona de extracción. Estos parámetros permitieron cuantificar y cualificar la estructura ósea. Las áreas de tejido de granulación, tejido óseo neoformado y médula ósea presentaron escasas diferencias a los 7 días de inicio de tratamiento, se observó presencia de hueso neoformado en el grupo $E(8 \%)$ que no presentan los otros grupos. Sin embargo, a los 15 días, Vitamina $\mathrm{E}$ registró valores de hueso neoformado por encima de todos los grupos experimentales ( $30 \%)$, con diferencias significativas con respecto al grupo Control $(p<0,05)$. A los 30 días se evidenciaron mayores valores de tejido óseo neoformado en el grupo de Vitamina E (88 \%) con respecto al grupo Control sin presentar diferencias significativas. El presente trabajo evidencia un aumento en el número de osteoblastos por $\mathrm{mm}$ lineal en los grupos $E$ y $A L+E$ con diferencias significativas con respecto a $C$ a los 30 días de tratamiento $(p<0,01)$; mostrando la misma diferencia significativa en la medición del espesor trabecular óseo. Esto indica que Vitamina $E$ favorece la actividad neoformadora de los osteoblastos que se expresa en un aumento de la can- 
tidad de tejido óseo nuevo. En los paramétros analizados AL no mostró diferencias significativas con el grupo C.

En concordancia con este trabajo, Jaime et al. (2005) no demostraron diferencias histológicas entre grupos tratados con AL de sodio tópico embebido en una esponja de colágeno a razón de $20 \mathrm{mg} / \mathrm{ml}$ y el grupo control. En cuanto al análisis histomorfométrico tampoco observaron diferencias significativas entre los grupos (Jaime et al.).

Por otro lado, varios informes han demostrado que $\mathrm{AL}$ puede afectar directamente la proliferación y diferenciación de osteoblastos. Komatsu et al. (2013) indican que AL aplicado localmente promueve la formación ósea mediante la estimulación de la proliferación y diferenciación de las células que forman el hueso adyacente a las superficies óseas en el modelo experimental, así como la inhibición de la función de los osteoclastos. Haddad et al. (2014) realizaron un análisis histomorfométrico de la matriz orgánica, observando que el tratamiento con $2 \mathrm{mg} / \mathrm{kg}$ de AL tuvo un efecto preventivo sobre la pérdida de masa ósea, ya que el área $\left(\mathrm{mm}^{3}\right)$ ocupada por la matriz orgánica fue mayor al grupo control $(p>0,05)$. El mecanismo de inhibición de los osteoclastos, producido por $\mathrm{AL}$, se ha atribuido a una disminución de la actividad celular, con efectos mínimos sobre el reclutamiento, la interferencia de receptores para las proteínas específicas de la matriz ósea y la producción de factor inhibidor de osteoclastos por los osteoblastos, lo que reduce la vida útil y/o el número de osteoclastos diferenciados, y la obstrucción de la resorción, al interferir en el margen rugoso de dichas células (Allen et al., 2010).

De la misma manera, hay estudios que determinaron que $A L$ se une a los cristales de apatita del hueso y que hay una acumulación de éste debajo del osteoclasto, haciendo que se reduzca la actividad cuando se altera el margen rizado, disminuyendo su superficie de resorción y de esta manera decreciendo la descalcificación focal realizada por los ácidos orgánicos. Al final del proceso se reduce el número de osteoclastos ya que se produce la apoptosis (Díaz et al., 2016).

Los efectos positivos de la vitamina $E$ en el hueso mediante la prevención del estrés oxidativo podrían ser mediados a través de una vía similar que implica el RANK / RANKL. Ha $\mathrm{H}$ et al. mostraron que la vitamina $E$ evitó la osteoclastogénesis y la resorción ósea mediante la supresión de RANKL expresión y señalización sin afectar a la expresión de OPG. También se ha demostrado que la vitamina $E$ inhibe la liberación y la expresión de citoquinas que resorben los huesos. Estos estudios sobre la acción de la vitamina E podrían sugerir que produce el reclutamiento de osteoclastos y la osteoclastogénesis (Ha et al.). Se necesitan estudios adicionales para determinar su mecanismo de acción exacto sobre el metabolismo óseo.

De acuerdo con los resultados del presente trabajo, Kasai et al. (2015) evaluaron los efectos de la Vitamina $E$ en los huesos en ratas normales alimentados con dietas que contenían diferentes niveles de aTocoferol, encontrando una tendencia a un aumento de la masa ósea dominante en la osteogénesis en el hueso esponjoso vertebral secundario, en el que se produce la remodelación ósea activa en los animales tratados con esta vitamina.

Así mismo, Hermizi et al. (2009) mostraron que la Vitamina $\mathrm{E}$ en dosis de $60 \mathrm{mg} / \mathrm{kg}$ de peso corporal durante dos meses fue capaz de mejorar el volumen óseo, el número trabecular, la tasa de aposición mineral y la tasa de formación ósea, y reducir el área de resorción en ratas ovariectomizadas.

Otros estudios difieren con estos resultados, encontrando que aT en dosis altas podría prevenir la osteoporosis en condiciones de estrés, pero podría ser perjudicial para el hueso en condiciones normales. Smith et al. (2005) utilizaron ratones machos de 8,5 meses de edad que recibieron diferentes dosis de aT (15 Ul/kg, $75 \mathrm{UI} / \mathrm{kg}$ y $500 \mathrm{IU} / \mathrm{kg}$ ); encontrando que los animales suplementados con la dosis de $500 \mathrm{UI} /$ $\mathrm{kg}$ de aT tenían un espesor trabecular y un volumen óseo significativamente menor en comparación con los animales suplementados con las dosis más bajas de aT. En otro estudio, Arjmandi et al. (2001) utilizaron ratones jóvenes (de 6 y 24 meses de edad) que fueron tratados con $30 \mathrm{mg} / \mathrm{kg}$ o $500 \mathrm{mg} / \mathrm{kg}$ de aT durante 30 días. Los ratones de mayor edad se beneficiaron con el tratamiento de alta dosis de aT, mostrando mejores propiedades biomecánicas del hueso. Estos cambios no se observaron en los ratones más jóvenes (Arjmandi et al.). Ambos estudios mencionados anteriormente podrían indicar que una dosis alta de aT puede no ejercer ningún efecto sobre el hueso normal, o incluso podría ser perjudicial, lo que se opone a los hallazgos del presente estudio.

En concordancia con esta investigación, un es- 
tudio utilizando ratones genéticamente incapaces de absorber Vitamina E, demostró disminución de masa ósea debido al aumento de la función de osteoclastos. Tras la alimentación con tocoferoles estos ratones restauraron un fenotipo de masa ósea normal y la función de osteoclastos disminuyó (Fujita et al., 2012). En una investigación con pérdida ósea inducida por ovariectomía, Vitamina $\mathrm{E}$ a $60 \mathrm{mg} / \mathrm{kg}$ por día y tocotrienol puro a la misma dosis fueron completamente protectores (Muhammad et al.).

\section{CONCLUSIONES}

En este trabajo, una dosis de Vitamina E de 20 $\mathrm{mg} / \mathrm{kg}$ de peso corporal administrada tres veces por semana demostró ser eficaz para mejorar las condiciones de regeneración ósea del alveolo postextracción del primer molar inferior en ratas macho, ya que histológicamente demostró una mejor organización del tejido a los 30 días, evidenciada por la presencia de tejido óseo compuesto; además produjo un aumento en el número de osteoblastos, por $\mathrm{mm}$ lineal $(13,4 \mathrm{~mm}$ a 30 días), presentando diferencias estadísticas con respecto al grupo Control $(p<0,01)$. Por otra parte, presentó un porcentaje mayor de espesor de trabéculas óseas $(26 \%)$ con respecto al grupo Control $(p<0,01)$. En cuanto a la administración infiltrativa de Alendronato, podemos concluir que mostró un comportamiento similar al Grupo Control, aunque con valores por encima del mismo. Esto podría deberse a su dificultad para difundir a través de las membranas celulares como consecuencia de la vía de administración utilizada.

La vía infiltrativa de acción local demostró ser una alternativa eficaz a las disponibles actualmente para la administración de fármacos, minimizando los efectos adversos sistémicos. Surge la necesidad de seguir ampliando estas investigaciones utilizando otras formas farmacéuticas que abrirán el horizonte para el diseño de nuevos fármacos y así poder incorporarlos al arsenal terapéutico en la práctica odontológica y sus distintas especialidades.

MORO, C.; AGUZZI, A. \& VIRGA, C. Evaluation of the infiltrative administration of vitamin $E$ and alendronate in the repair of alveoli post-extraction in rats. Int. J. Odontostomat., 13(4):402-410, 2019.

ABSTRACT: Bisphosphonates (BP) decrease bone resorption to curb the activity of the osteoclasts. Vitamin $E$ is an antioxidant and its positive effect on the bone would be by preventing oxidative stress. Infiltrative Alendronate and vitamin $\mathrm{E}$ administration wasstudied to determine if they favored the formation of bone in bone repair of the postextraction alveolus. Male Wistar rats were used $(n=96), 90$ $\pm 15 \mathrm{~g}$, underwent extraction of the lower first molars. They were divided into 4 groups: A control group (C) received saline. The Group at the $0.5 \mathrm{mg} / \mathrm{Kg}$; Group E received 20 $\mathrm{mg} / \mathrm{kg}$; and combined treatment group to $\mathrm{AL}$ and $\mathrm{E}$. The animals were sacrificed at days $0,7,15$ and 30 post extraction. With the resection of the jaws; samples were decalcified with EDTA and then included in paraffin. Histological cuts were made and colored with Hematoxylin/ eosin. Histomorphometric and histological analysis was performed. We used analysis of variance (ANOVA). In the histological analysis, 7 to 15 days the Group E presented greater neoformation of bone tissue than other groups. At 30 days mature bone was observed, with presence of osteons in the Group E. Study shows significant differences in the number of osteoblast histomorphometric function to 15 to 30 days by linear $\mathrm{mm}$, among the group to the $+E$ and $C(p<$ 0.01 ) and 30-day difference was found among the Group $E$ and $C(p<0.01)$. When measuring thick trabecular, significant differences were observed at 30 days between the $A L+E$ and C Group $(p<0.01)$ and between $C$ and $E(p<0.01)$. Vitamin $E$ showed that administered infiltrative favors the bone remodeling in post extraction sockets.

KEY WORDS: osteogenesis, alendronate, vitamin $\mathrm{E}$

\section{REFERENCIAS BIBLIOGRÁFICAS}

Aimetti, M.; Romano, F.; Griga, F. B. \& Godio, L. Clinical and histologic healing of human extraction sockets filled with calcium sulfate. Int. J. Oral Maxillofac. Implants, 24(5):902-9, 2009.

Ali-Erdem, M.; Burak-Cankaya, A.; Cemil-Isler, S.; Demircan, S.; Soluk, M.; Kasapoglu, C. \& Korhan-Oral, C. Extraction socket healing in rats treated with bisphosphonate: animal model for bisphosphonate related osteonecrosis of jaws in multiple myeloma patients. Med. Oral Patol. Oral Cir. Bucal, 16(7):e87983, 2011.

Allen, M. R.; Erickson, A. M.; Wang, X.; Burr, D. B.; Martin, R. B. \& Hazelwood, S. J. Morphological assessment of basic multicellular unit resorption parameters in dogs shows additional mechanisms of bisphosphonate effects on bone. Calcif. Tissue Int., 86(1):6771, 2010.

Arjmandi, B. H.; Akhter, M.; Chakkalakal, D.; Khalil, D. A.; Lucas, E. A. \& Juma, S. Effects of isoflavones, vitamin E, and their combination on bone in an aged rat model of osteopenia. J. Bone Miner. Res., 16(Suppl. 1):s533, 2001.

Azzi, A.; Gysin, R.; Kempná, P.; Ricciarelli, R.; Villacorta, L.; Visarius, T. \& Zingg, J. M. The role of alpha-tocopherol in preventing disease: from epidemiology to molecular events. Mol. Aspects Med., 24(6):325-36, 2003.

Barone, A.; Ricci, M.; Tonelli, P.; Santini, S. \& Covani, U. Tissue changes of extraction sockets in humans: a comparison of spontaneous healing vs. ridge preservation with secondary soft tissue healing. Clin. Oral Implants Res., 24(11):1231-7, 2013. 
Devlin, H.; Hoyland, J.; Newall, J. F. \& Ayad, S. Trabecular bone formation in the healing of the rodent molar tooth extraction socket. J. Bone Miner. Res., 12(12):2061-7, 1997.

Díaz, D. H.; Rodas, J. A.; Bozzini, C. E.; Mandalunis, P. M. \& Escudero, N. D. Sequential administration of alendronate and strontium ranelate: histomorphometry and bone biomechanics in ovariectomized animals. Acta Odontol. Latinoam., 29(2):16877,2016

Fontana, S. Respuesta Biológica de los Rellenos Óseos. Estudio Experimental. Tesis Doctoral. Córdoba, Facultad de Odontología, Universidad Nacional de Córdoba, 2009.

Fujita, K.; Iwasaki, M.; Ochi, H.; Fukuda, T.; Ma, C.; Miyamoto, T.; Takitani, K.; Negishi-Koga, T.; Sunamura, S.; Kodama, T.; et al. Vitamin $\mathrm{E}$ decreases bone mass by stimulating osteoclast fusion. Nat. Med., 18(4):589-94, 2012.

Guglielmotti, M. B. \& Cabrini, R. L. Alveolar wound healing and ridge remodeling after tooth extraction in the rat: a histologic, radiographic, and histometric study. J. Oral Maxillofac. Surg., 43(5):359-64, 1985.

Ha, H.; Lee, J. H.; Kim, H. N. \& Lee, Z. H. a-Tocotrienol inhibits osteoclastic bone resorption by suppressing RANKL expression and signaling and bone resorbing activity. Biochem. Biophys. Res. Commun., 406(4):546-51, 2011.

Haddad, P. T.; Salazar, M. \& Hernandes, L. Histomorphometry of the organic matrix of the femur in ovariectomized rats treated with sodium alendronate. Rev. Bras. Ortop., 50(1):100-4, 2014.

Hermizi, H.; Faizah, O.; Ima-Nirwana, S.; Ahmad Nazrun, S. \& Norazlina, M. Beneficial effects of tocotrienol and tocopherol on bone histomorphometric parameters in sprague-dawley male rats after nicotine cessation. Calcif Tissue Int., 84(1):65-74, 2009.

Jaime, A.; Anbinder, A.; Lima, A. P.; Balducci, I. \& Rocha, R. The influence of local delivery of alendronate sodium on regeneration of calvarial defects in ovariectomized rats. Cienc. Odontol. Bras., 8(2):70-9, 2005.

Kasai, S.; Ito, A.; Shindo, K.; Toyoshi, T. \& Bando, M. High-dose atocopherol supplementation does not induce bone loss in normal rats. PLoS One, 10(7):e0132059, 2015.

Komatsu, K.; Shimada, A.; Shibata, T.; Wada, S.; Ideno, H.; Nakashima, K.; Amizuka, N.; Noda, M. \& Nifuji, A. Alendronate promotes bone formation by inhibiting protein prenylation in osteoblasts in rat tooth replantation model. J. Endocrinol., 219(2):145-58, 2013.

Muhammad, N.; Luke, D. A.; Shuid, A. N.; Mohamed, N. \& Soelaiman, I. N. Two different isomers of vitamin e prevent bone loss in postmenopausal osteoporosis rat model. Evid. Based Complement. Alternat. Med., 2012:161527, 2012.

Roberts, W. E.; Turley, P. K.; Brezniak, N. \& Fielder, P. J. Implants: Bone physiology and metabolism. CDA J., 15(10):54-61, 1987.

Rogers, M. J. New insights into the molecular mechanisms of action of bisphosphonates. Curr. Pharm. Des., 9(32):2643-58, 2003.

Smith, B. J.; Lucas, E. A.; Turner, R. T.; Evans, G. L.; Lerner, M. R.; Brackett, D. J.; Stoecker, B. J. \& Arjmandi, B. H. Vitamin E provides protection for bone in mature hindlimb unloaded male rats. Calcif. Tissue Int., 76(4):272-9, 2005.

Thompson, K.; Rogers, M. J.; Coxon, F. P. \& Crockett, J. C. Cytosolic entry of bisphosphonate drugs requires acidification of vesicles after fluid-phase endocytosis. Mol. Pharmacol., 69(5):1624-32, 2006.

Ting, K.; Petropulos, L. A.; Iwatsuki, M. \& Nishimura, I. Altered cartilage phenotype expressed during intramembranous bone formation. J. Bone Miner. Res., 8(11):1377-87, 1993.

Weibel, E. R.; Kistler, G. S. \& Scherle, W. F. Practical stereological methods for morphometric cytology. J. Cell Biol., 30(1):23-38, 1966.
Dirección para correspondencia:

Dra. Alejandra Aguzzi

Cátedra de Farmacología y Terapéutica A

Facultad de Odontología

Universidad Nacional de Córdoba

ARGENTINA

Email: aleceagu@yahoo.com.ar

Recibido : 03-05-2019

Aceptado: 09-07-2019 